\title{
Electromagneto-Mechanical Coupling Analysis of a Test Module in J-TEXT Tokamak During Plasma Disruption
}

\author{
Haijie Dong ${ }^{\mathrm{a}}$, Zhensheng Yuan ${ }^{\mathrm{a}}$, Hongwei Yuan ${ }^{\mathrm{a}}$, Zhenmao Chen $^{\mathrm{a}^{*}}$, Jinhong Yang ${ }^{\mathrm{b}}$, Weihua Wang ${ }^{\mathrm{b}}$ \\ ${ }^{a}$ State Key Laboratory for Strength and Vibration of Mechanical Structures, \\ Shanxi Engineering Research Center for NDT and Structural Integrity Evaluation \\ $X i$ 'an Jiaotong University, Xi'an 710049, China \\ ${ }^{b}$ Institute of Applied Physics of AOA, Hefei 230031, China
}

\begin{abstract}
In this paper, the dynamic response during plasma disruption of a test blanket module in vacuum vessel (VV) of the Joint TEXT (J-TEXT), which is an experimental Tokamak device with iron core, was simulated by applying a program developed by authors on the ANSYS platform using its parametric design language (APDL). The moving coordinate method as well as the load transfer and sequential coupling strategy were adopted to cope with the electromagneto-mechanical coupling effect. To establish the numerical model, the influence of the iron core on the eddy current and electromagnetic (EM) force during disruption was numerically investigated at first and the influence was found not significant. Together with the geometrical features of the J-TEXT Tokamak structure, $180^{\circ}$ sector models without magnetic core were finally established for the EM field and the structural response simulations. To obtain the source plasma current, the plasma current evolution during disruption was simulated by using the Tokamak Simulation Code (TSC). With the numerical models and the source plasma current, the dynamic response of both the VV structure and the test module were calculated. The numerical results show that the maximum stress of the test module is in safe range, and the magnetic damping effect can weaken vibration of the test module. In addition, simulation without considering the coupling effect was carried out, which shows that the influence of coupling effect is not significant for the peak stress of the J-TEXT disruption problem.
\end{abstract}

Keywords: Test module, J-TEXT Tokamak, APDL, Plasma disruption, Electromagneto-mechanical coupling effect, Moving coordinate method.

\section{Introduction}

To measure the electromagnetic (EM) loads on the blanket module in Tokamak during plasma disruption and to validate the related numerical simulation tools, a cuboidal test module was designed and fabricated for disruption testing in the Joint TEXT (J-TEXT) Tokamak which was formerly known as the TEXT/ TEXT-U Tokamak [1]. The test module is set in a window of the vacuum vessel (VV) of the J-TEXT Tokamak with a specially designed fixture. During disruption, large EM forces caused by the eddy current and the confinement magnetic field may act on the in-vessel conducting structures [2] and cause significant structural deformation and vibration. The deformation and vibration of the VV structure and the test module, in turn, may also affect the EM field environment and EM forces. Therefore, the electromagneto-mechanical coupling effect is a key issue to improve the simulation accuracy of EM loads and the corresponding structural dynamic response [3] for in-vessel components of Tokamak structures.

To deal with the coupling effect, velocity induced electric field term $\mathbf{v} \times \mathbf{B}$ (where $\mathbf{v}$ is velocity and $\mathbf{B}$ is magnetic flux density) is commonly adopted to take the interaction between EM fields and the structural vibration into account [4]. However, after adding the velocity term into governing equations of eddy current problem, the finite element (FE) coefficient matrices for eddy current analysis becomes asymmetrical, which significantly increases the computational burden to solve the eddy current problem and even may worsen its numerical stability [5]. To overcome these problems, the moving coordinate approach, which can treat the coupling behaviors of magnetic damping and magnetic stiffness effects at the same time, was proposed in [5]. An integral formula based on the Lagrangian coordinate system [6] was developed to solve the nonlinear eddy current problem of deformable bodies without using the velocity term explicitly, and numerical model for the solution of the classic electromagneto-mechanical coupled problem was presented in [7]. Authors [8,9] have proposed a numerical method counting on the Maxwell equations in Lagrangian description and A- $\phi$ formulation to address the eddy current problem of a deformable body. However, there is still no result reported yet about the application of the moving coordinate approach to electromagneto-mechanical coupling analysis of practical Tokamak structures.

In this paper, in addition to the EM loads on the VV and test module, the structural deformation and vibration were calculated for the J-TEXT Tokamak structure by applying a program developed by authors on the ANSYS platform. Moving coordinate method as well as the load transfer and sequential coupling strategy were employed to deal with the electromagneto-mechanical coupling effect. Both calculations with and without consideration of the coupling effect were carried out to investigate the influence of coupling effect on the J-TEXT Tokamak application. 


\section{Method for Coupling Analysis}

Load transfer and step by step sequential coupling strategy, which need both EM and mechanical numerical models, are usually adopted to treat the influence of the coupling effect between the EM fields and the structural response in the transient dynamic response simulation. Therefore, an EM model and a mechanical numerical model have to be established respectively, which are usually modeled with different FE meshes. For sequential coupling analysis, the EM fields and structural response are calculated separately, and the coupling effect is considered through load transfers, i.e., EM loads on structures are obtained from EM field calculation and transferred to the mechanical model as input loads, while the solved mechanical displacement and velocity are transferred to the EM model to initiate the EM field calculation of the next time step.

For conventional method, governing equations of EM fields are formulated in an Eulerian framework, which means governing equations of given coordinate system. In this case, velocity term $\mathbf{v} \times \mathbf{B}$ appears explicitly in the governing equations of the eddy current problem. On the other hand, the governing equations of the Lagrangian description do not include the velocity term explicitly, i.e., the governing equations are just the same with those for static rigid structures [8]. With variables in the Lagrangian description, however, Lagrangian meshes or so called co-moving meshes are necessary in simulations. In co-moving meshes, the mesh points remain coincident with the material points and elements deform with the material. The co-moving meshes can be realized by using the moving coordinate strategy, i.e., to update the FE mesh at each time step based on the deformed structure during vibration.

In practice, the ANSYS software offers so called "mesh morphing" commands, which meets requirement to adopt the moving coordinate strategy in practical simulation. Moreover, ANSYS parametric design language (APDL) is also convenient to manipulate the flow of calculations and data to realize the sequential coupling of eddy current and vibration. Based on these considerations, authors have developed a numerical code with the APDL in ANSYS platform for simulating eddy current of a moving elastic body [9]. As shown in Fig.1, the FE mesh for eddy current calculation is updated at each integration time step based on the structural deformation of the previous time step, i.e., the coordinates of each node are modified during each time step of simulation. The validity of the moving coordinate strategy has been proved by solving the TEAM 16 benchmark problem with our code and comparing the results with those of the conventional coupling method and the experiments [9]. The very good agreement of the simulation and experimental results shown in Fig. 2 reveals that the moving coordinate strategy is very efficient for the dynamic response analysis considering electromagneto-mechanical coupling effect. As the governing equations are just the same with those of the conventional eddy current problem, the moving coordinate method is easy to be implemented for treating coupling problem of large scale based on the commercial software such as ANSYS.

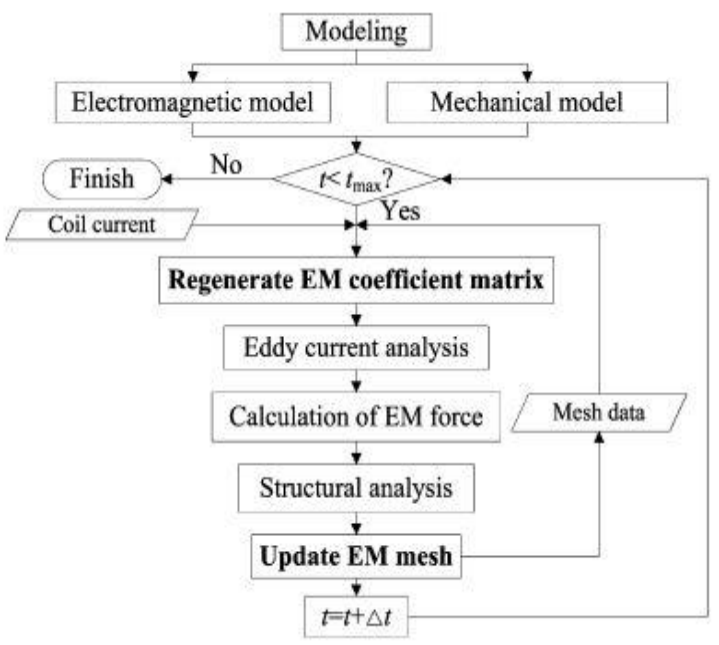

Fig. 1. Flowchart of coupled analysis method [9]

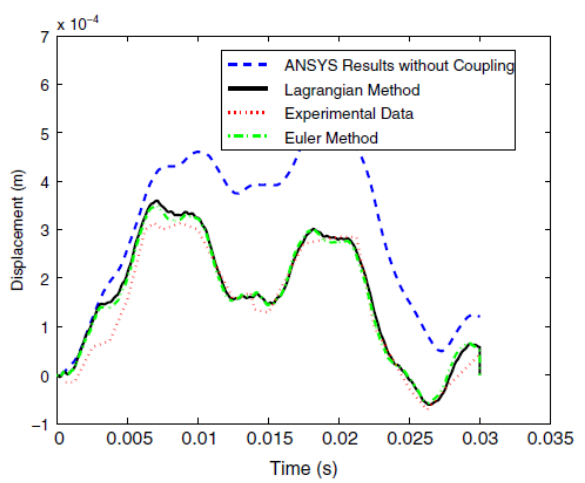

Fig. 2. Displacement versus time for point $A, B_{y}=0.1 T$ [9]

In accordance with moving coordinate method, the magnetic damping effect is taken into account through eddy current analysis of deformable bodies and the magnetic stiffness effect is considered by EM mesh morphing, i.e., the moving coordinate method takes both the electromagneto-mechanical coupling effect of magnetic damping and magnetic stiffness into account at the same time. This is also an advantage of this strategy comparing with the conventional coupling method, as the magnetic stiffness matrix is usually difficult to obtain. Based on these considerations, the APDL program developed by authors is adopted for the coupling dynamic response simulations in the following sections.

\section{Numerical Modeling}

\subsection{Model simplification}

\subsubsection{Influence of the iron magnetic core}

Different from many other Tokamak structures, the JTEXT Tokamak has an iron core which makes the simulation of EM fields complicated. To investigate the influence of the iron core on EM fields, an EM numerical model including iron core, vacuum vessel $(\mathrm{VV})$, toroidal field (TF) coils, poloidal field (PF) coils, filamentary plasma currents and test module was established according to the practical geometric parameters of J-TEXT Tokamak by using ANSYS 
commercial software. The relative permeability of core material depends on the magnetic field inside the core nonlinearly, which may affect the eddy current of the whole system. To check the influence of the core, the eddy currents were solved respectively for core relative permeability of $1,10,20,50,100,200,500$ and 1,000 by using the established EM numerical model. A pulsed plasma current was adopted as the excitation in the simulations.

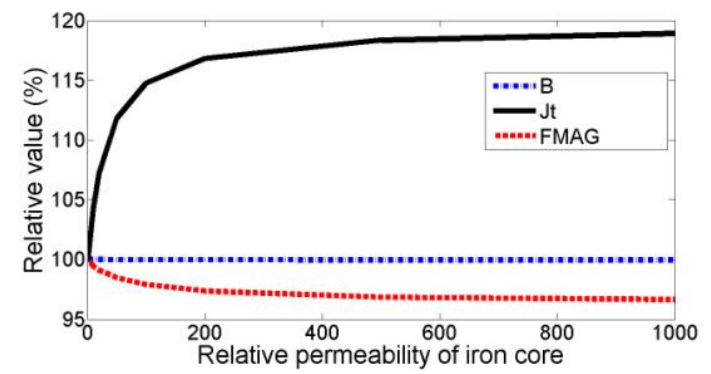

Fig. 3. Influence of permeability on simulation results.

As the eddy currents are relatively large at time point of $15 \mathrm{~ms}$ for present problem, the numerical results at this time instant, including magnetic flux density (B), eddy current density ( $\mathrm{Jt}$ ) and magnetic force density (FMAG), at the center of an element located in the test module surface layer were extracted and shown in Fig.3. The results for unit relative permeability correspond to the case without iron core and were used to normalize the values shown in Fig.3. From Fig.3, one can find that the influence of iron core on the EM calculation results is less than $20 \%$ for all the fields. In present work, as the major aim is to demonstrate the validity of the moving coordinate coupling method for a practical Tokamak structure, and to check the influence of electromagnetomechanical coupling effect on the dynamic response during plasma disruption, it is reasonable to ignore the iron core in the EM numerical model of J-TEXT to improve simulation efficiency.

\subsubsection{Model reduction using symmetry conditions}

The VV of J-TEXT Tokamak is composed of two semi-ring parts connected by two keystones and are insulated each other. Hence, a $180^{\circ}$ sector model can be used to further improve the simulation efficiency. To verify the validity of this $180^{\circ}$ sector reduced model, both simulations employing reduced and full EM models were carried out.

The cyclic symmetric condition was applied to the EM model by coupling all DOFs of nodes using the "CPCYC" command of APDL. To implement the cyclic symmetric condition, nodes on one side of the model should completely correspond to those on the other side. Hence, areas on the $-45^{\circ}$ side (the position of the test module is taken as $0^{\circ}$ ) were meshed with mesh200 elements, which were ignored in the practical calculation. The mesh of this part was copied to the areas on the $135^{\circ}$ side (see Fig. 4). The flux parallel condition was considered at the nodes laying on the central $z$ axis and the surfaces of the model. In addition, the conductivity of insulation layers was set as zero to consider the insulation between semi-ring parts.

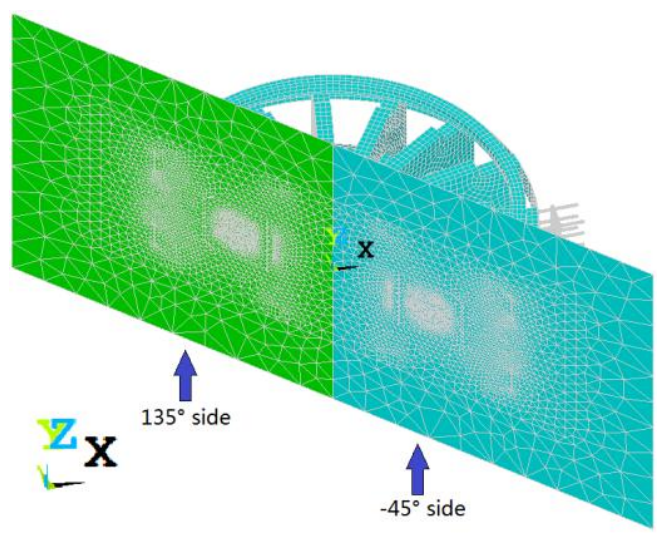

Fig. 4. Mesh200 elements on both sides of the half EM model.

Fig. 5 and Fig. 6 show the comparisons of magnetic flux density and eddy current density of the same element between the results of the half and full EM models. As shown in these figures, results of the $180^{\circ}$ sector reduced model agree well with those of the full model. This reveals that the $180^{\circ}$ sector reduced model and the corresponding boundary condition treatment are correct. Therefore, the $180^{\circ}$ sector reduced model without iron core will be adopted in the following practical simulations.

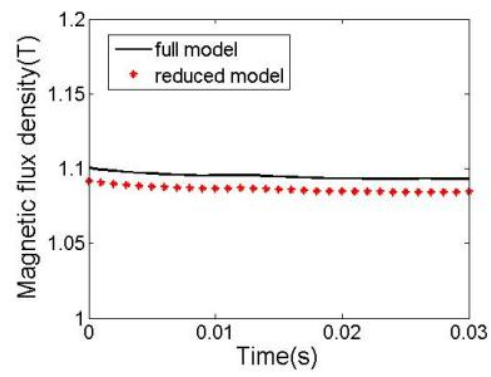

Fig. 5. Comparison of magnetic flux density between reduced and full EM models without iron core.

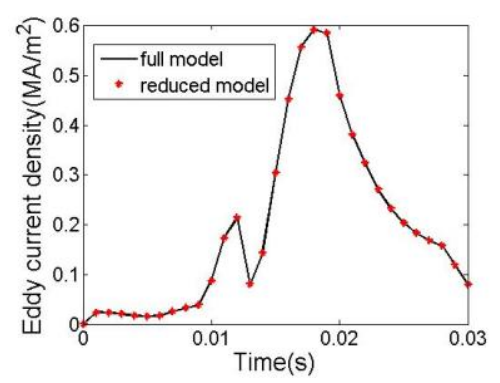

Fig. 6. Comparison of eddy current density between reduced and full EM models without iron core.

\subsection{Final numerical models}

\subsubsection{EM model}

As shown in Fig. 7, the $180^{\circ}$ sector reduced EM analysis model without iron core is composed of VV, TF coils, PF coils, filamentary plasma currents, test module and its supporting components. Fig. 7 also shows the test module is assembled in a window of the $\mathrm{VV}$ with supporting components. The cuboidal box shaped test module is of $316 \mathrm{~L}$ stainless steel and of plate structure of $220 \mathrm{~mm}, 210 \mathrm{~mm}$ and $280 \mathrm{~mm}$ size in radial, toroidal 
and poloidal direction respectively. The thickness of the module plate is $30 \mathrm{~mm}$. The test module is fixed to the VV with a fixture of tube beam of the same $316 \mathrm{~L}$ stainless material.

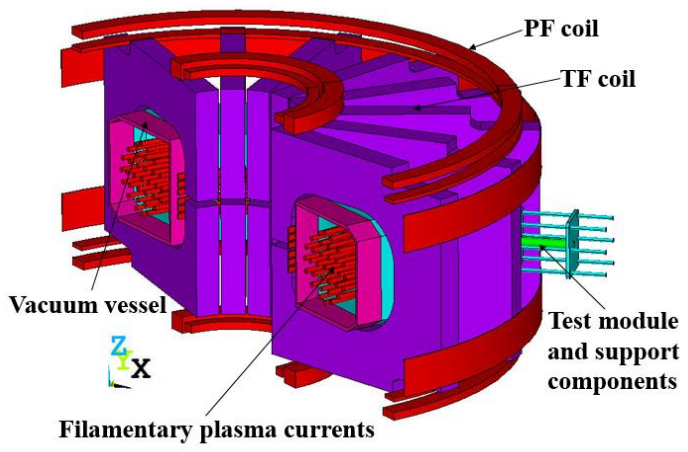

Fig. 7. Configuration of $180^{\circ}$ sector reduced EM geometry modeling without iron core.

The completed 3D CAD model (Fig.7) was imported to ANSYS and then meshed. The SOLID97 element was adopted for mesh divisions of all the conducting structures, coils, air and plasma currents. Totally 190,347 nodes and 1,021,520 elements were generated for the $180^{\circ}$ sector EM analysis model.

The same treatment of boundary conditions shown in section 3.1.2 was also adopted. Though there is only one test module in practical experiment, the $180^{\circ}$ sector reduced model was used as the test module is relative small comparing with the VV structure that means very small influence on the EM fields at the other side of VV.

The current on PF and TF coils were used to generate confinement magnetic fields. Time dependent current density was employed on each PF coil while a constant current value of $600 \mathrm{kA}$ was applied in all the TF coils.

\subsubsection{Modeling of plasma current}

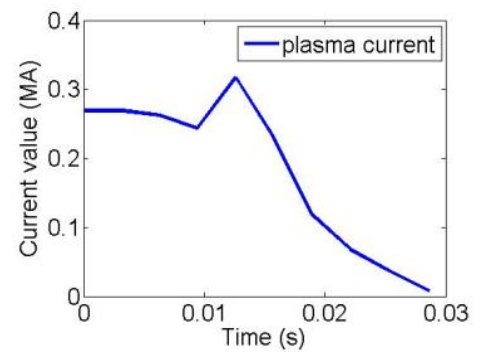

Fig. 8. Total plasma current versus time during disruption.

The plasma current during disruption was simulated by the Tokamak Simulation Code (TSC) which is an axisymmetric two-dimensional time dependent simulation code capable to solve the magnetohydrodynamic (MHD) equations describing the transport time-scale evolution of magnetized Tokamak plasma [8]. The evolution of plasma current during major disruption was approximated by 33 circular filamentary currents distributed uniformly in the cross-section of plasma current area. The time-varying current densities of each current filament were extracted from the 2D transient plasma current, which was obtained from the TSC simulation. The time evolution of the total plasma current during plasma disruption is shown in Fig.8.

\subsubsection{Mechanical model}

The mechanical numerical model only consists of the conducting structures including $\mathrm{VV}$, test module and its supporting components. The SOLID95 element was employed to discretize those structures. Total 26,985 nodes and 80,097 elements were produced for the $180^{\circ}$ sector mechanical model.

The material of $\mathrm{VV}$, supporting plate and module room is 304 stainless steel while the test module and support rod are made of 316L stainless steel.

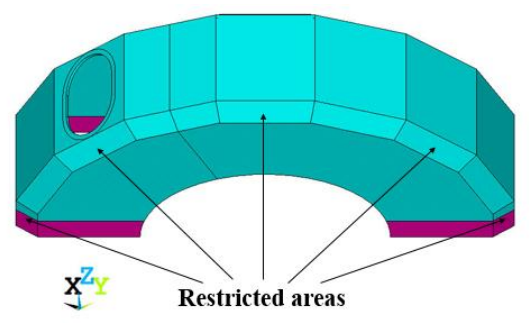

Fig. 9. Restricted areas of VV.

The symmetric condition was utilized on the mechanical model. As shown in Fig. 9, at the nodes located on these restricted areas, all three DOFs were restrained using the " $\mathrm{D}$ " command of ANSYS internal functionality. EM loads transferred from the calculation of EM fields were applied on nodes.

\section{Results and Discussions}

\subsection{Simulation procedure}

The electromagneto-mechanical coupling analysis was carried out by using the APDL program of moving coordinate method described in section 2 to calculate the deformation and vibration of test module and its support components during the plasma disruption.

To apply the moving coordinate method to our simulation, the air domain nearby structures were selected after the mechanical simulation at each time step, and were morphed using the "DVMORPH" command of APDL to make the mesh points remain coincident with the material points and elements deformed with the structures. Before the EM simulation at next time step, the FE coefficient matrices were regenerated as the elements have deformed with structures.

Relative commands were used in our APDL program to implement the load transfers between the EM and mechanical analyses. At each time step, the EM load data were written into a file after the EM simulation and "LDREAD" command was employed to read the load information from the data file before the mechanical calculation. Since the corner nodes of elements in structural regions were completely corresponding to each other between these two models, the load transfer could be accomplished without any interpolation. 
Several trials have been done by choosing the time step length as $0.1 \mathrm{~ms}, 0.2 \mathrm{~ms}, 0.3 \mathrm{~ms}$ and $0.4 \mathrm{~ms}$ respectively to determine the proper time step length for EM and vibration simulations. It was found that the displacement response curves of the test module were not smooth enough for $0.3 \mathrm{~ms}$ and $0.4 \mathrm{~ms}$ time step length. However, such situation was improved if the time step was reduced to $0.2 \mathrm{~ms}$. Furthermore, no obvious difference except lower computational efficiency was found when the time step length was further changed to $0.1 \mathrm{~ms}$. Therefore, the time step length was finally chosen as $0.2 \mathrm{~ms}$ in practical simulation. Besides, the total simulation time range was 0 to $250 \mathrm{~ms}$.

\subsection{Typical results}

The eddy current distribution in the VV at $15 \mathrm{~ms}$ time instant is shown in Fig.10. The eddy current forms loops between the current in the inner part (near central $z$ axis) and the outer part of VV, i.e., the currents in the inner part and in the outer part of $\mathrm{VV}$ are in opposite direction. This is reasonable due to presence of the insulation layers between two semi-ring parts. On the right side of Fig.10, the vector arrows are denser since smaller and irregular elements were generated to cope with the window and test module.

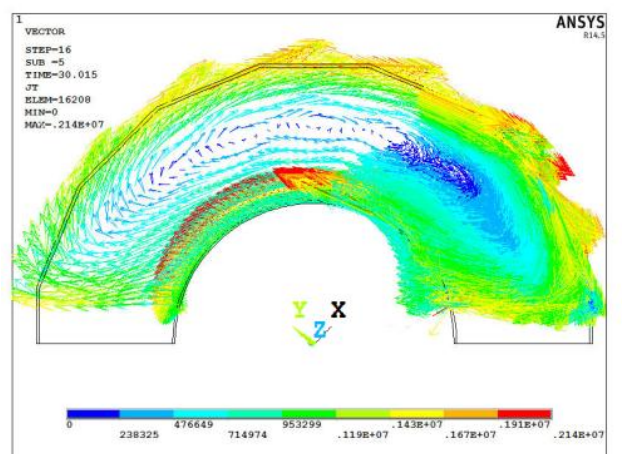

Fig. 10. Vector graphics of eddy current distribution on VV.

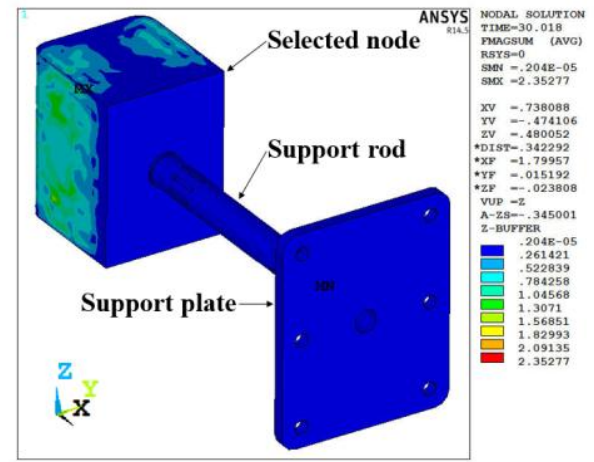

Fig.11. Distribution of EM loads on test module and support components at $18 \mathrm{~ms}$ (units: $\mathrm{N}$ ).

For the test module, the EM loads caused by the interaction of induced eddy current and magnetic fields are mainly acted on the two side surfaces of the module as shown in Fig. 11. Such EM loads made the test module, supporting rod and supporting plate deform and vibrate. The supporting rod subjected to bending and torsional moments was the major stressed component.
Non-coupling analysis was also carried out to check the influence of coupling effect. Based on the simulation results, though the maximum EM loads took place at 18 $\mathrm{ms}$ of the disruption, the maximum values of structural stress and strain occurred at about $26 \mathrm{~ms}$ time instant. Fig. 12 to Fig. 15 show the distribution of $1^{\text {st }}$ principal stress and elastic strain in the module at the moment of $26 \mathrm{~ms}$ in both the coupled and uncoupled cases. In Fig. 12 and Fig. 13, we can see that the maximum structural stress values of coupling analysis and non-coupling analysis are $74.3 \mathrm{MPa}$ and $75.9 \mathrm{MPa}$ respectively at the end of the supporting rod, which are much smaller than the yield stress of $316 \mathrm{~L}$ stainless steel, and indicate structural safety during the selected major plasma disruption of J-TEXT. Fig. 14 shows the maximum strain $(329 \mu \varepsilon)$ of coupling analysis case and Fig. 15 displays that the maximum strain value of non-coupling analysis is $336 \mu \varepsilon$. From these results, one can find that the maximum values of structural stress and strain of coupling analysis are only a little smaller than those of non-coupling analysis, but the difference is not significant in the considered cases.

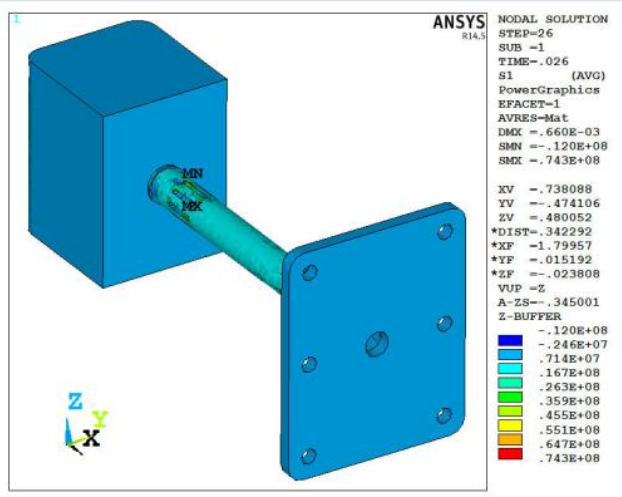

Fig.12. Distribution of $1^{\text {st }}$ principal stress on test module and support components at $26 \mathrm{~ms}$ (coupling).

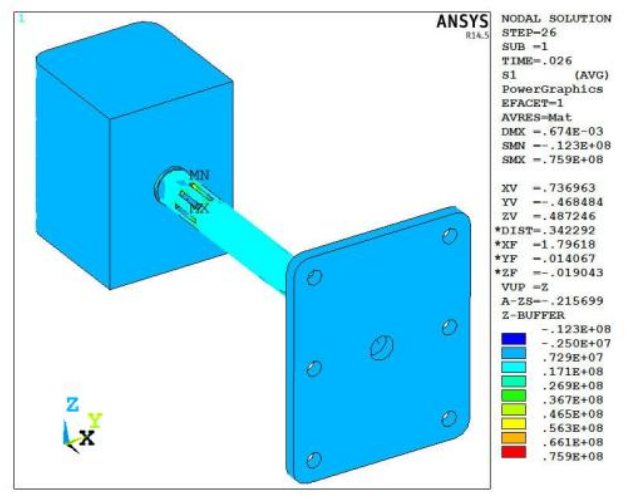

Fig.13. Distribution of 1st principal stress on test module and support components at $26 \mathrm{~ms}$ (non-coupling).

To view the vibration status of the test module structures, a node located at the corner of test module (see Fig. 11) was selected as the monitoring point. The displacement in poloidal direction at the selected node is illustrated in Fig. 16. The vibration amplitude of the selected node without considering the coupling effect is almost the same with that just after the disruption. However, for the case with coupling effect, the vibration decays rapidly with time (dash line of Fig.16). This result reveals that the vibration of structures can be 
significantly affected by the electromagneto-mechanical coupling effect, or in other words, the magnetic damping effect can weaken the vibration of in-vessel components. On the other hand, the vibration frequency is almost the same for these two cases that means the magnetic stiffness effect is small for this nonmagnetic test module.

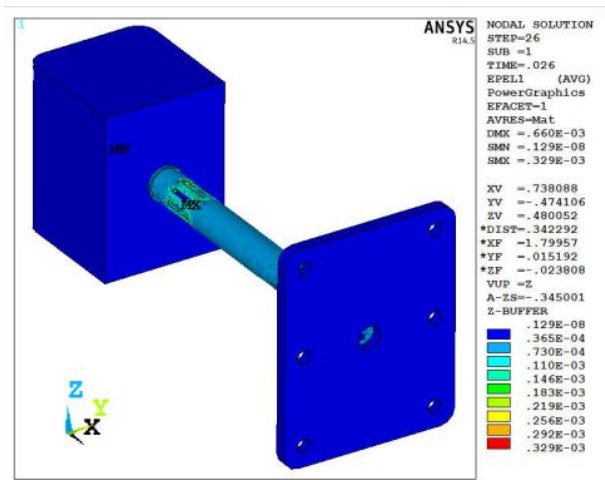

Fig. 14. Distribution of $1^{\text {st }}$ principal elastic strain on test module and support components at $26 \mathrm{~ms}$ (coupling).

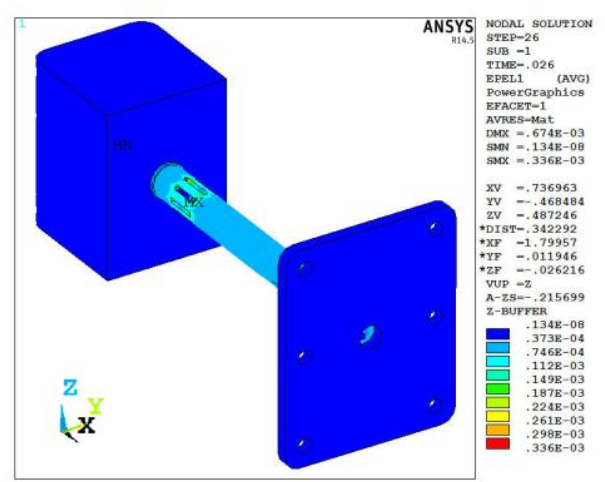

Fig. 15. Distribution of 1st principal elastic strain on test module and support components at $26 \mathrm{~ms}$ (non-coupling).

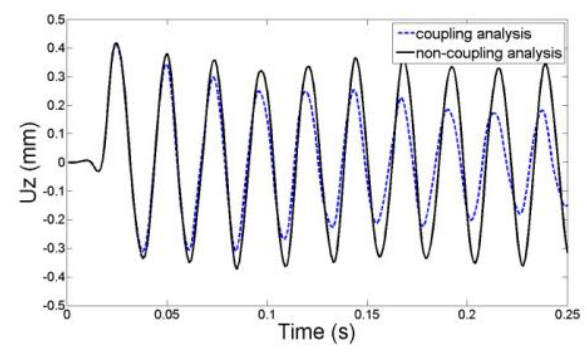

Fig. 16. Comparison of time history of displacement in $z$ direction at a selected node.

\section{Conclusions}

In this paper, the moving coordinate coupling method was applied to simulate the dynamic response of the test module set in the VV of the J-TEXT Tokamak during its major plasma disruption with consideration of the electromagneto-mechanical coupling effect of both magnetic damping and magnetic stiffness. The plasma current during disruption was simulated by using TSC and was equivalent to current filaments as transient current source for coupling vibration simulation. A $180^{\circ}$ sector reduced model without considering the iron core was adopted for both the EM and mechanical response simulations. Numerical results showed that the moving coordinate method and the corresponding APDL code were suitable for the simulation of a practical Tokamak structure. It was found that the maximum stress was at the end of the support rod of the module structures during disruption, and the value of maximum stress was 74.3 $\mathrm{MPa}$ which was much smaller than the yield stress of $316 \mathrm{~L}$ stainless steel. Comparisons of the results of coupled and non-coupled analyses also showed that the influence of the coupling effect was not significant on the maximum stress and strain during disruption for $\mathrm{J}$ TEXT problem. However, the vibration amplitude of structures could be significantly affected by the magnetic damping force.

\section{Acknowledgments}

The authors would like to thank the National Magnetic Confinement Fusion Program of China (2013GB113005), National Science Foundation of China (No. 51277139, 11321062 and 51407132) and National Basic Research Program of China (No.2011CB610303) for funding this study.

\section{References}

[1] G. Zhuang et al., The reconstruction and research progress of the TEXT-U Tokamak in China, Nuclear Fusion, 51 (2011) 094020.

[2] R. Albanese et al., Effects of asymmetric vertical disruptions on ITER components, Fusion Engineering and Design, 94 (2015) 7-21.

[3] T. Jordan, Disruption induced mechanical loads calculated with a coupled 3-D eddy current/2-D MHD plasma model, Fusion Engineering and Design, 43 (1998) 173-187.

[4] T. Takagi and J. Tani, Dynamic behavior analysis of a plate in magnetic field by full coupling and MMD methods, IEEE Transactions on Magnetics, 30 (5) (1994) 3296-3299.

[5] S. Kurz et al., A novel formulation for 3D eddy current problems with moving bodies using a Lagrangian description and BEM-FEM coupling, IEEE Transactions on Magnetics, 34 (5) (1998) 3068-3073.

[6] R. Albanese et al., A nonlinear eddy-current integral formulation for moving bodies, IEEE Transactions on Magnetics, 34 (5) (1994) 2529-2534.

[7] A. Portone et al., Numerical model of the dynamic response of $3 \mathrm{D}$ conducting structures with magnetic damping, presented at Compumag 2015, June 28-July 2, Montreal, Canada.

[8] W. Li et al., A moving coordinate numerical method for analysis of electromagneto-mechanical coupled behavior of structure in strong magnetic field aiming at application to Tokamak structures, Plasma Science and Technology, 16 (12) (2014) 1163-1170.

[9] W. Li et al., Numerical analysis of electromagnetomechanical coupling using lagrangian approach and adaptive time steeping method, International Journal of Applied Mechanics, 6 (5) (2014) 1450051.

[10] S. Jardin et al., TSC simulation of ohmic discharges in TFTR, Nuclear Fusion, 33 (3) (1993) 371-382. 